2011

\title{
Ducted kinetic Alfvén waves in plasma with steep density gradients
}

Saeid Houshmandyar

Earl E. Scime

Follow this and additional works at: https://researchrepository.wvu.edu/faculty_publications

\section{Digital Commons Citation}

Houshmandyar, Saeid and Scime, Earl E., "Ducted kinetic Alfvén waves in plasma with steep density gradients" (2011). Faculty Scholarship. 135.

https://researchrepository.wvu.edu/faculty_publications/135

This Article is brought to you for free and open access by The Research Repository @ WVU. It has been accepted for inclusion in Faculty Scholarship by an authorized administrator of The Research Repository @ WVU. For more information, please contact ian.harmon@mail.wvu.edu. 


\title{
Ducted kinetic Alfvén waves in plasma with steep density gradients
}

\author{
Saeid Houshmandyar ${ }^{1,2}$ and Earl E. Scime ${ }^{2}$ \\ ${ }^{1}$ Solar Observatory Department, Prairie View A\&M University, Prairie View, Texas 77446, USA \\ ${ }^{2}$ Department of Physics, West Virginia University, Morgantown, West Virginia 26506-6315, USA
}

(Received 2 September 2011; accepted 27 October 2011; published online 29 November 2011)

Given their high plasma density $\left(n \sim 10^{13} \mathrm{~cm}^{-3}\right)$, it is theoretically possible to excite Alfvén waves in a conventional, moderate length $(L \sim 2 \mathrm{~m})$ helicon plasma source. However, helicon plasmas are decidedly inhomogeneous, having a steep radial density gradient, and typically have a significant background neutral pressure. The inhomogeneity introduces regions of kinetic and inertial Alfvén wave propagation. Ion-neutral and electron-neutral collisions alter the Alfvén wave dispersion characteristics. Here, we present the measurements of propagating kinetic Alfvén waves in helium helicon plasma. The measured wave dispersion is well fit with a kinetic model that includes the effects of ion-neutral damping and that assumes the high density plasma core defines the radial extent of the wave propagation region. The measured wave amplitude versus plasma radius is consistent with the pile up of wave magnetic energy at the boundary between the kinetic and inertial regime regions. (C) 2011 American Institute of Physics. [doi:10.1063/1.3662113]

\section{INTRODUCTION}

Alfvén waves are the fundamental, low-frequency, electromagnetic modes of magnetized plasmas. In the ideal magnetohydrodynamic (MHD) approximation, they propagate exclusively along the background magnetic field at the Alfvén speed $\mathrm{v}_{\mathrm{A}}=B_{0} /\left(\mu_{0} n_{i} m_{i}\right)^{1 / 2}$, where $B_{0}$ is the background magnetic field strength and $n_{i}$ and $m_{i}$ are the ion density and mass, respectively. More realistic descriptions of the plasma environment require inclusion of finite plasma pressure, electron inertia, collisions with background neutrals, spatial gradients in the magnetic field and plasma density, and boundaries. These additional effects complicate the simplistic model of Alfvén propagation by introducing new phenomena such as nonzero parallel electric fields and cross-field propagation.

In low resistivity plasmas such as the solar atmosphere, where the plasma and magnetic fields are strongly coupled, driven plasma flows can lead to the excitation of Alfvén waves. Recent observations from Hinode, a high-resolution solar imaging mission, ${ }^{1}$ confirm that upward propagating Alfvén waves in the solar chromosphere exist and appear to be driven by photospheric flows and upwelling plasma. ${ }^{2}$ The solar corona, the region above the chromosphere, is nearly 200 times hotter than the Sun's surface. Therefore, the Hinode observations have lent support to models of coronal heating which hypothesize that low-frequency Alfvén waves can heat ions in the corona if the waves propagate into the regions of weaker magnetic field strength (through an ion cyclotron resonance) or through damping of Alfvén wave cascades at ion spatial scales. The amount of energy stored in the observed Alfvén waves is consistent with the magnitude of the calculated additional energy source required to explain the million degree coronal temperatures. Prior to the Hinode measurements, the observations of Alfvén waves in the high speed solar wind ${ }^{3}$ triggered the development of Alfvén wave-based coronal ion heating models. $^{4-7}$ Although the coronal ion heating problem has motivated the development of many recent models of Alfvén wave propagation, dispersive Alfvén waves, i.e., those Alfvén waves with nonzero parallel electric fields and which propagate obliquely, have been of long standing theoretical and experimental interest because of their possible roles in auroral phenomena ${ }^{8,9}$ and fusion plasmas.

First, formulated by Hannes Alfvén for a conducting fluid, ${ }^{10}$ the dispersion relation for a classic shear Alfvén waves (SAW) is $\omega=k_{\|} \mathrm{v}_{\mathrm{A}}$, where $k_{\|}$is the parallel component of the wave number, and parallel and perpendicular directions are defined with respect to the background magnetic field $\mathbf{B}_{\mathbf{0}}$. A classic SAW has a large perpendicular wavelength $\left(\lambda_{\perp}\right)$ and insignificant parallel electric field. It also has a constant parallel phase velocity $\left(\mathrm{v}_{\text {Phase } \|}=\omega / k_{\|}=\mathrm{v}_{\mathrm{A}}\right)$. However, when $\lambda_{\perp}$ becomes small, the wave dispersion changes significantly and additional effects must be included in the theory. Just how small is "small" for the perpendicular wavelength depends on the ability of the electrons to respond to the wave fields. When the electron thermal speed exceeds the Alfvén speed, $\mathrm{v}_{T e}=\left(2 k_{B} T_{e} / m_{e}\right)^{1 / 2}>\mathrm{v}_{A}$, electrons move fast enough to respond adiabatically to the wave fields and the wave is commonly referred to as a "kinetic Alfvén wave (KAW)." In this limit, the spatial scale that defines a "small" perpendicular wavelength is the ion sound gyroradius, $\rho_{s}=c_{s} / \omega_{c i}$, where $\omega_{c i}=e B_{0} / m_{i} c$ is the ion cyclotron frequency and $c_{s}=\left(k_{B} T_{e} / m_{i}\right)^{1 / 2}$ is the ion sound speed, with $e$ being the charge of an electron, $k_{B}$ is Boltzmann's constant, $m_{e}$ is the electron mass, and $c$ is the speed of light. ${ }^{11}$

For electron thermal speeds less than the Alfvén speed, electron inertia becomes important and the wave is referred to as an "inertial Alfvén wave (IAW)." In this limit, the relevant "small" spatial scale is the electron skin depth, $\delta_{e}=c / \omega_{p e}$, where $\omega_{p e}=\left(4 \pi n_{e} e^{2} / m_{e}\right)^{1 / 2}$ and $n_{e}$ is the electron density. The electron thermal to Alfvén speed ratio can also be expressed as the plasma beta scaled by the ion-toelectron mass ratio,

$$
\left(\frac{\mathrm{v}_{T e}}{\mathrm{v}_{A}}\right)^{2}=\bar{\beta}=\frac{m_{i}}{m_{e}} \beta=\left(\frac{m_{i}}{m_{e}}\right)\left(\frac{8 \pi n_{e} k_{B} T_{e}}{B_{0}^{2}}\right) .
$$


Thus, very low beta plasmas, such as the auroral zone, the edge of laboratory plasmas, and the base of the solar corona, are systems in which inertial Alfvén waves propagate. In higher beta systems, e.g., fusion plasmas, the solar wind, and the magnetosphere, kinetic Alfvén wave propagation is expected. Of particular importance to the experiments that are described in this work is that KAWs are forward propagating (phase and group velocities are in the same direction) waves, whereas IAWs are backward propagating waves. Therefore, a KAW wave propagating into a region of decreasing plasma density, at a density gradient for instance, will have to couple into IAW propagating in the opposite direction; essentially trapping the wave power on the higher density side of the gradient.

The low frequency nature of Alfvén waves (i.e., $\omega / \omega_{c i}<1$, required to satisfy the constraint of the MHD approximation) and the range of Alfvén speeds achievable in laboratory experiments typically necessitate a very long experimental system in which to study Alfvén wave physics. The long parallel length $(L \sim 20 \mathrm{~m})$ and reproducible plasma of the large plasma device (LAPD) ${ }^{12}$ have made it an ideal facility for Alfvén wave experiments. The higher densities of helicon plasma sources, as with some previous facilities used for Alfvén wave studies, ${ }^{13,14}$ can theoretically compensate for the much shorter achievable plasma lengths. ${ }^{15,16}$ However, there are a number of challenges arising from the characteristics of helicon sources which need to be addressed. One is the steep radial gradient in plasma density, which imposes two distinct regimes of kinetic and inertial Alfvén wave propagation. The second challenge is the high neutral to plasma density fraction, which introduces wave damping through finite ion-neutral and electron-ion collisions.

This work presents laboratory observations of low frequency wave excitation and propagation in a "helicon" plasma source, which are consistent with the expected propagation of "kinetic Alfvén waves." The measured dispersion relation is in good agreement with a KAW model that includes the effects of neutral collisions and finite perpendicular wave number $\left(k_{\perp}\right)$ effects. In Sec. II, we provide the relevant theoretical background for the experimental conditions. The experimental apparatus and diagnostic tools are presented in Sec. III. The measurements and analysis and interpretation of the measurements are provided in Secs. IV and $\mathrm{V}$.

\section{ALFVÉN WAVE THEORY}

Finite perpendicular wavelength effects in Alfvén wave propagation typically arise in laboratory experiments through the excitation of Alfvén waves by small scale antennas, e.g., the shear Alfvén wave experiments in the LAPD device, ${ }^{17}$ or through the effects of chamber walls, which introduce a finite perpendicular wavelength by forcing the wave fields of the Alfvén waves to satisfy conducting or insulating wall boundary conditions. ${ }^{18}$ Regardless of the origin of the finite perpendicular wavelength, its existence modifies the simple Alfvén wave dispersion relation. In the experiments reported here, we will demonstrate that the observed Alfvén wave propagation is consistent with the imposition of a finite perpendicular wavelength by boundary conditions created by the high density "core" region typical of a helicon plasma.

Another limitation of the classic Alfvén model approach is the assumption of extremely low wave frequencies. Typical laboratory studies excite waves at frequencies comparable to the ion-cyclotron frequency, $f_{\mathrm{ci}}=\omega_{c i} / 2 \pi$, because it is experimentally easier to couple significant powers to antennas at higher frequencies and because the physics of wave propagation near the ion cyclotron resonance is often of interest. Although inclusion of the Hall term in the MHD momentum equation introduces a finite frequency term in the dispersion relation, ${ }^{19}$ other finite frequency effects and critical phenomena such as Landau damping and finite Larmor radius corrections are not easily incorporated in a MHD formulation. ${ }^{20}$ Thus, we briefly review here the warm plasma theory model for Alfvén wave propagation.

In a warm plasma, the ion and electron particle distribution functions evolve according to the Maxwell-Boltzmann equation

$$
\frac{\partial f}{\partial t}+\mathbf{v} \cdot \nabla f+\frac{q}{m}[\mathbf{E}+\mathbf{v} \times \mathbf{B}] \cdot \nabla_{\mathrm{v}} f=\left.\frac{d f}{d t}\right|_{c},
$$

where the distribution function is $f(\mathbf{x}, \mathbf{v}, t)=f_{0}(\mathbf{v})+f_{1}(\mathbf{x}, \mathbf{v}, t)$ and $f_{0}$ is the equilibrium distribution function (assumed to be Maxwellian). The right hand side of Eq. (2) is the collision operator and for collisionless plasmas $(\mathrm{d} f / \mathrm{d} t)_{c}=0$. The field perturbations are given by Maxwell's equations and satisfy the wave equation

$$
\mathbf{k} \times(\mathbf{k} \times \mathbf{E})+\frac{\omega^{2}}{c^{2}} \overleftrightarrow{\boldsymbol{\varepsilon}} \cdot \mathbf{E}=0,
$$

where $c$ is the speed of light and $\overrightarrow{\boldsymbol{\varepsilon}}$ is the warm plasma dielectric tensor for a wave frequency of $\omega$. The elements of the warm plasma dielectric tensor for collisionless plasmas are readily available in the literature, e.g., Swanson. ${ }^{21}$ In the low frequency limit and assuming an equilibrium magnetic field along the $z$ axis, Eq. (3) reduces to ${ }^{22}$

$$
\left(n_{\|}^{2}-\varepsilon_{x x}\right) \varepsilon_{z z}+n_{\perp}^{2} \varepsilon_{x x}=0,
$$

where $n_{\|}=c k_{\|} / \omega, n_{\perp}=c k_{\perp} / \omega$, and the relevant $\overrightarrow{\boldsymbol{\varepsilon}}$ tensor elements are

$$
\begin{aligned}
\varepsilon_{x x} & =1+\sum_{j} \frac{\omega_{p j}^{2}}{\sqrt{2} \omega k_{\|} \mathrm{v}_{T j}} \frac{1}{\mu_{j}} \sum_{n=-\infty}^{\infty} n^{2} \Gamma_{n}\left(\mu_{j}\right) Z\left(\zeta_{n j}\right) \\
& \approx \frac{\omega_{p i}^{2}}{\omega_{c i}^{2}-\omega^{2}} \frac{1-\Gamma_{0}\left(\mu_{i}\right)}{\mu_{i}} \\
\varepsilon_{z z} & =1-\sum_{j} \frac{\omega_{p j}^{2}}{\sqrt{2} \omega k_{\|} \mathbf{v}_{T j}} \sum_{n=-\infty}^{\infty} \Gamma_{n}\left(\mu_{j}\right) Z^{\prime}\left(\zeta_{n j}\right) \\
& \approx 2 \frac{\omega_{p e}^{2}}{\sqrt{2} \omega k_{\|} \mathbf{v}_{T e}} \zeta_{0 e}\left[1+\zeta_{0 e} Z\left(\zeta_{0 e}\right)\right],
\end{aligned}
$$

where $j$ denotes the species ( $e$ for electron and $i$ for ions), $\mu_{j}=k_{\perp}^{2} \rho_{j}^{2}, \rho_{j}$ is the gyroradius for each species, $\Gamma_{n}\left(\mu_{j}\right)$ $=\exp \left(-\mu_{j}\right) I_{n}\left(\mu_{j}\right), I_{n}(x)$ is the modified Bessel function, $Z\left(\zeta_{n j}\right)$ is the plasma dispersion function ${ }^{20}$ with an argument of 


$$
\zeta_{n j}=\frac{\omega-n \omega_{c j}}{\sqrt{2} \omega k_{\|} \mathbf{v}_{T j}}
$$

and $Z^{\prime}\left(\zeta_{n j}\right)=-2\left[1+\zeta_{n j} Z\left(\zeta_{n j}\right)\right]$ is the derivative of the plasma dispersion function with respect to $\zeta_{n j}$. Employing the analysis of Gekelman et al., ${ }^{17}$ the low frequency Alfvén wave dispersion relation is

$$
Z^{\prime}\left(\zeta_{0 e}\right)\left[\frac{1}{\bar{\beta}} \frac{1-\left(\omega / \omega_{c i}\right)^{2} \mu_{i}}{1-\Gamma_{0}\left(\mu_{i}\right)}-\zeta_{0 e}^{2}\right]=\left(\frac{c k_{\perp}}{\omega}\right)^{2}
$$

Equation (8) incorporates finite Larmor radius and finite frequency effects through the $1-\Gamma_{0}\left(\mu_{i}\right)$ and $1-\left(\omega / \omega_{c i}\right)^{2}$ terms, respectively.

For low ion temperatures relative to the electron temperature and in the kinetic regime, where $\bar{\beta}>1, Z^{\prime}$ approaches a constant value of -2 and the dispersion relation becomes ${ }^{17}$

$$
\omega=k_{\|} \mathbf{v}_{A} \sqrt{1-\omega^{2} / \omega_{c i}^{2}+k_{\perp}^{2} \rho_{s}^{2}}
$$

Note that the ion sound gyroradius scale appears naturally in the dispersion and the finite frequency effect is equally apparent. Note also that with these effects included, the Alfvén waves become dispersive, i.e., the phase speed and group velocity now depend on the perpendicular wavelength of the wave. For small perpendicular structures, $k_{\perp}^{2} \rho_{s}^{2} \geq 1$, the parallel phase velocity of the wave can exceed $\mathrm{v}_{\mathrm{A}}$. In our helicon source plasmas, the ion sound gyroradius is typically $0.9 \mathrm{~cm}$. Thus, perpendicular wavelengths on the order of $7 \mathrm{~cm}$ can have a significant effect on the wave dispersion. In the limit of small wave frequencies relative to the ion cyclotron frequency, the common expression for a dispersive kinetic Alfvén wave is recovered. ${ }^{20}$

$$
\omega=k_{\|} \mathrm{v}_{A} \sqrt{1+k_{\perp}^{2} \rho_{s}^{2}} .
$$

In the inertial regime (low density, low temperature, and strong magnetic field), where $\bar{\beta}<1, Z^{\prime}$ is approximately equal to $\zeta^{-2}$ and the dispersion relation becomes ${ }^{17}$

$$
\omega=k_{\|} \mathrm{v}_{A} \sqrt{\frac{1-\omega^{2} / \omega_{c i}^{2}}{1+k_{\perp}^{2} \delta_{e}^{2}}} .
$$

Here again, in the low frequency limit, the more common expression for dispersive inertial Alfvén waves is recovered

$$
\omega=\frac{k_{\|} \mathrm{v}_{A}}{\sqrt{1+k_{\perp}^{2} \delta_{e}^{2}}} .
$$

The inertial and kinetic dispersion relations both reduce to the classic shear Alfvén wave expression in the limit of zero perpendicular wave number $\left(k_{\perp} \rho_{s} \ll 1\right.$ or $\left.k_{\perp} \delta_{e} \ll 1\right)$ and very low frequency waves. For the inertial waves, the electron skin depth is typically $0.2 \mathrm{~cm}$ in our plasmas. Thus, for low frequency waves in the inertial regime, perpendicular wavelengths less than $35 \mathrm{~cm}$ will result in a decrease of the wave phase speed to values less than $\mathrm{v}_{\mathrm{A}}$.
The plasma environment created in laboratory experiments also requires an assessment of the impact of electronion and ion-neutral collisions on Alfvén wave propagation. Collisions become important when the electron mean free path becomes comparable to the Alfvén wave wavelength. Gekelman et al. showed that electron-ion collision frequencies as large as 50 times the ion cyclotron frequency (in their afterglow plasma) did not significantly affect the wave propagation characteristics. ${ }^{17}$ For Coulomb collision rates, in cgs units, given by ${ }^{23}$

$$
v_{e i}=\frac{\pi n_{e} e^{4} \ln \Lambda}{\sqrt{2 m_{e} k_{B} T_{e}^{3}}},
$$

where $\ln \Lambda$ is the Coulomb logarithm, they argued that the principal effect was pitch angle scattering of the electrons and that the pitch angle scattering actually served to reduce Landau damping of the waves by reducing the length of time that particles could remain in Landau resonance with the wave. For our helicon plasmas, the electron-ion collision rates are comparable to those in the LAPD afterglow plasmas. Thus, Alfvén wave propagation should not be suppressed by Coulomb collisions.

An important difference, however, is the much larger ion-neutral collision frequency in the bulk helicon plasma. Whereas the LAPD plasmas are nearly fully ionized, the helium helicon plasma ionization fraction in these experiments varies from $50 \%-90 \%$ in the plasma core to $10 \%$ or less at the plasma edge. The effects of neutral collisions on Alfvén wave propagation was first considered by Woods. ${ }^{18}$ Later researchers have extended his initial work. ${ }^{24,25}$ Woods showed that the effect of ion-neutral collision can be incorporated into the Alfvén wave dispersion through a pseudoion-cyclotron frequency $\omega_{c i}^{*}=\omega_{c i} / S$, where $S$ is a frequency dependent and complex factor representing mass loading of ions by neutrals and it describes the complex coupling between the plasma and neutral fluids,

$$
S=1+\frac{\rho_{n} / \rho_{m}}{1-i \omega / \omega_{n i}},
$$

where $\rho_{m}$ and $\rho_{n}$ are the plasma and neutral mass densities, respectively, and $\omega_{n i}=n_{n} \sigma_{n i} \bar{u}_{i}$ is the ion-neutral collision frequency, with $n_{n}$ being the neutral atom density, $\bar{u}_{i}$ the average ion thermal velocity, and $\sigma_{n i}$ the ion-neutral collision cross section. For a helium plasma, the ion-neutral collision cross section (including charge exchange and elastic collisions) $)^{26}$ is approximately $6.8 \times 10^{-15} \mathrm{~cm}^{2}$ for an ion temperature of $0.25 \mathrm{eV}$.

The ion-neutral collision effect is introduced into the Alfvén wave expressions, Eqs. (9) and (11), through an effective ion mass $m_{i}^{*}=m_{i} S$. The most significant impact of ion-neutral collisions is the downshift and of the ioncyclotron resonance in the Alfvén dispersion relation to frequencies below the ion cyclotron frequency (collisions can also suppress the resonance). The downshift has been observed experimentally and has been reproduced in more complete theoretical models. ${ }^{15,27}$ For helium plasma parameters of $n_{e}=1 \times 10^{13} \mathrm{~cm}^{-3}, B_{0}=560 \mathrm{G}$, and $T_{i}=0.25 \mathrm{eV}$, the 
effect of ion-neutral collisions on the Alfvén dispersion relation is shown in Fig. 1, where Eq. (9) is plotted as a function of the ratio of neutral to plasma density for waves with zero perpendicular wave number.

\section{EXPERIMENTAL APPARATUS}

The Hot hELicon eXperiment (HELIX) ${ }^{28}$ is a typical linear helicon source with a hybrid stainless steel-Pyrex ${ }^{\mathrm{TM}}$ vacuum chamber (Fig. 2); the Pyrex ${ }^{\mathrm{TM}}$ chamber is $61 \mathrm{~cm}$ long and $10 \mathrm{~cm}$ in diameter and the stainless steel section is $91 \mathrm{~cm}$ long and $15 \mathrm{~cm}$ in diameter. One set of four $6 \mathrm{in}$. Conflat ${ }^{\mathrm{TM}}$ ports and four sets of four $2-3 / 4$ in. Conflat ${ }^{\mathrm{TM}}$ crossing ports are used for diagnostics access. The stainless steel chamber opens into the $1.8 \mathrm{~m}$ diameter, $4.4 \mathrm{~m}$ long diffusion LEIA (large experiment on instabilities and anisotropies) chamber. All the measurements reported here were performed in the HELIX portion of the system. A $19 \mathrm{~cm}, m$ $=+1$ helical antenna is wrapped around the Pyrex ${ }^{\mathrm{TM}}$ tube and couples the rf power supplied from a $2 \mathrm{~kW}$, ENI 1000, rf amplifier into the plasma. The antenna system operates over a frequency range of 6-18 MHz. The power is impedancematched through a $\pi$-matching network consisting one load and three tuning capacitors. Ten water-cooled electromagnets provide a constant and nearly uniform magnetic field of $0-1400 \mathrm{G}$. The base pressure of the HELIX-LEIA system is maintained at $10^{-7}$ Torr by three turbomolecular drag pumps. A MKS 1179 mass flow valve with a PR-4000 flow controller governs the flow of gas into HELIX. The gas pressure is measured by a Balzers PKR250 full range pressure gauge located at far end of the HELIX.

For a HELIX magnetic field $\left(B_{\mathrm{H}}\right)$ of $560 \mathrm{G}$ (corresponding to a helium ion-cyclotron frequency of $213.6 \mathrm{kHz}$ ), zero LEIA magnetic field $\left(B_{\mathrm{L}}\right)$, an antenna frequency of 13.56 $\mathrm{MHz}, 900 \mathrm{~W}$ of rf power, and a helium discharge pressure of 18 mTorr, the mean electron density $\left(n_{e}\right)$ and temperature

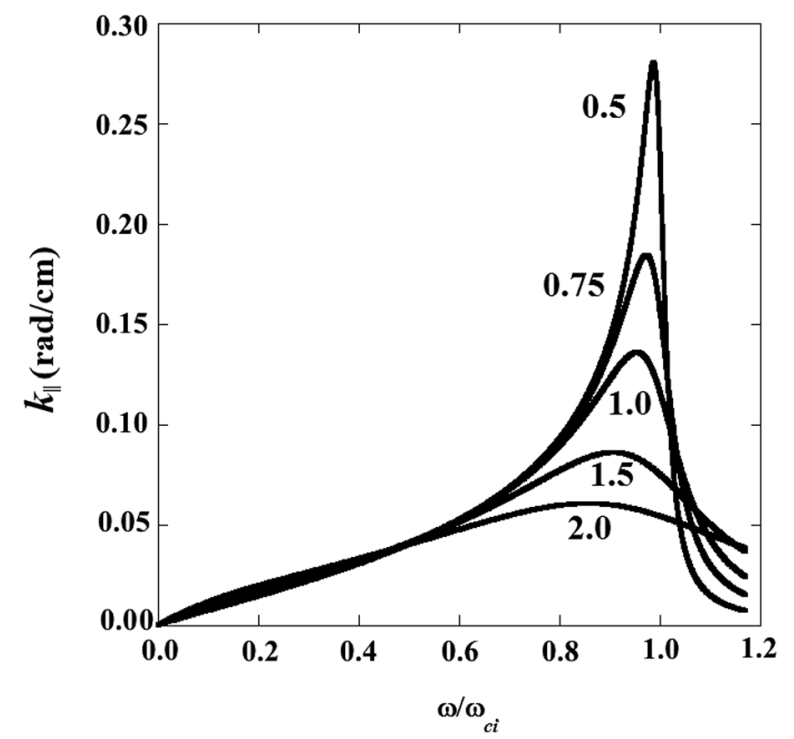

FIG. 1. The parallel wave number versus normalized wave frequency for shear Alfven waves according to Eq. (9); assuming a zero perpendicular wave number. Dispersion curves are shown for neutral to ion density ratios of $0.5,0.75,1.0,1.5$, and 2 . The plasma parameters used in the calculation are $n_{e}=1 \times 10^{13} \mathrm{~cm}^{-3}, T_{i}=0.25$, and $B_{0}=560 \mathrm{G}$.

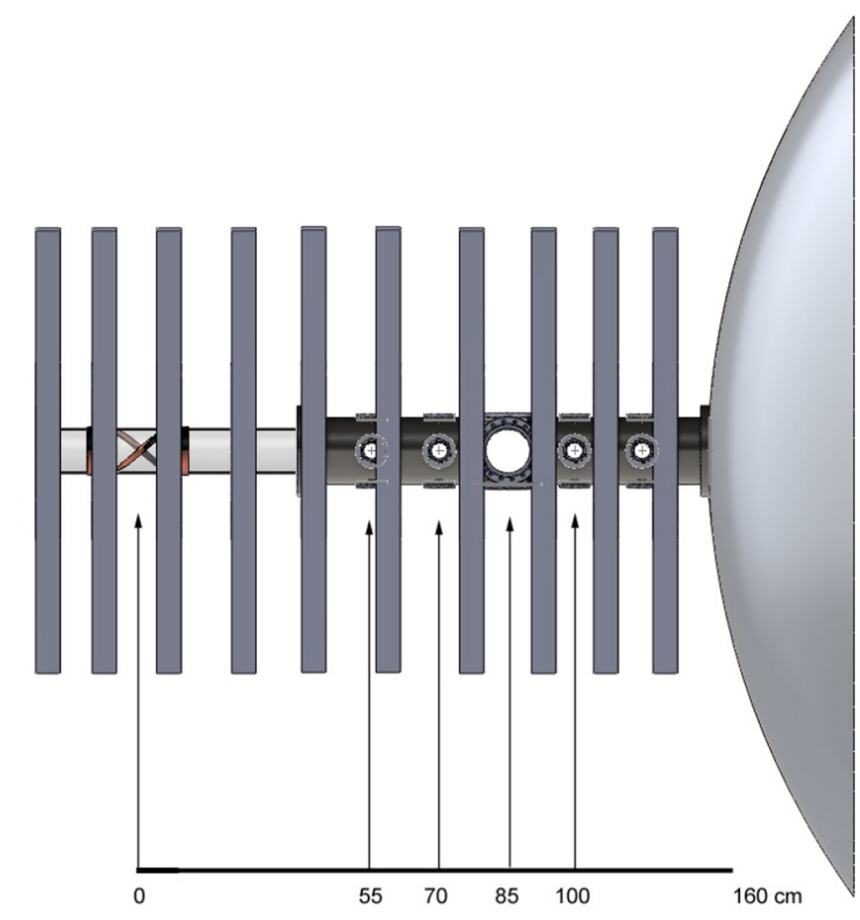

FIG. 2. (Color online) Schematic of HELIX-LEIA apparatus (a portion of the end of the LEIA chamber is shown at the right of this figure). The $19 \mathrm{~cm}$ long $m=1$ antenna surrounds the glass portion of the chamber at $z=0 \mathrm{~cm}$. Ten electromagnets, aligned as shown, provide the magnetic field in the source region. The ports at 70,85 , and $100 \mathrm{~cm}$ were used for electrostatic and magnetic fluctuation measurements. The launching antenna was positioned at $z=55 \mathrm{~cm}$.

$\left(T_{e}\right)$ as a function of axial and radial position are shown in Fig. 3. The gas was fed into the system from the end of HELIX opposite of the LEIA chamber. ${ }^{29,30}$ The measurements shown in Fig. 3 include a linear interpolation to fill the locations in the plot between the actual measurements. The measured values are preserved in the contour plot at each measurement location. Also shown in the Fig. 3 are the ion sound gyroradius and a comparison between the measured electron thermal and Alfvén speeds as a function of axial and radial positions. The electron temperature and density measurements are obtained with a moveable rf-compensated Langmuir probe. These source parameters are chosen to maximize the plasma density while simultaneously minimizing the on-axis neutral density (as determined by laser absorption spectroscopy ${ }^{31}$ ); the measured neutral density is $\sim 5.0 \times 10^{12} \mathrm{~cm}^{-3}$ out to a radius of $\sim 4 \mathrm{~cm}$, yielding an ionization fraction of $50 \%$. The electrostatic and/or magnetic fluctuation measurements reported here are performed at axial locations of $z=70 \mathrm{~cm}, z=85 \mathrm{~cm}$, and $z=100 \mathrm{~cm}$.

The waves are launched from a dipole antenna located at $z=55 \mathrm{~cm}$ (Fig. 4). The antenna consists of 4 turns of 4 gauge, enamel insulated, copper wire encased in a ceramic annulus. For wave launching, the antenna is positioned at the center of the plasma and waves excited such that the magnetic perturbation is parallel to the background magnetic field, i.e., a compressional Alfvén wave should be excited. The antenna design was chosen to minimize perturbation to the plasma by letting the high-density center of plasma pass through the $1.52 \mathrm{~cm}$ diameter hole in the center of the 

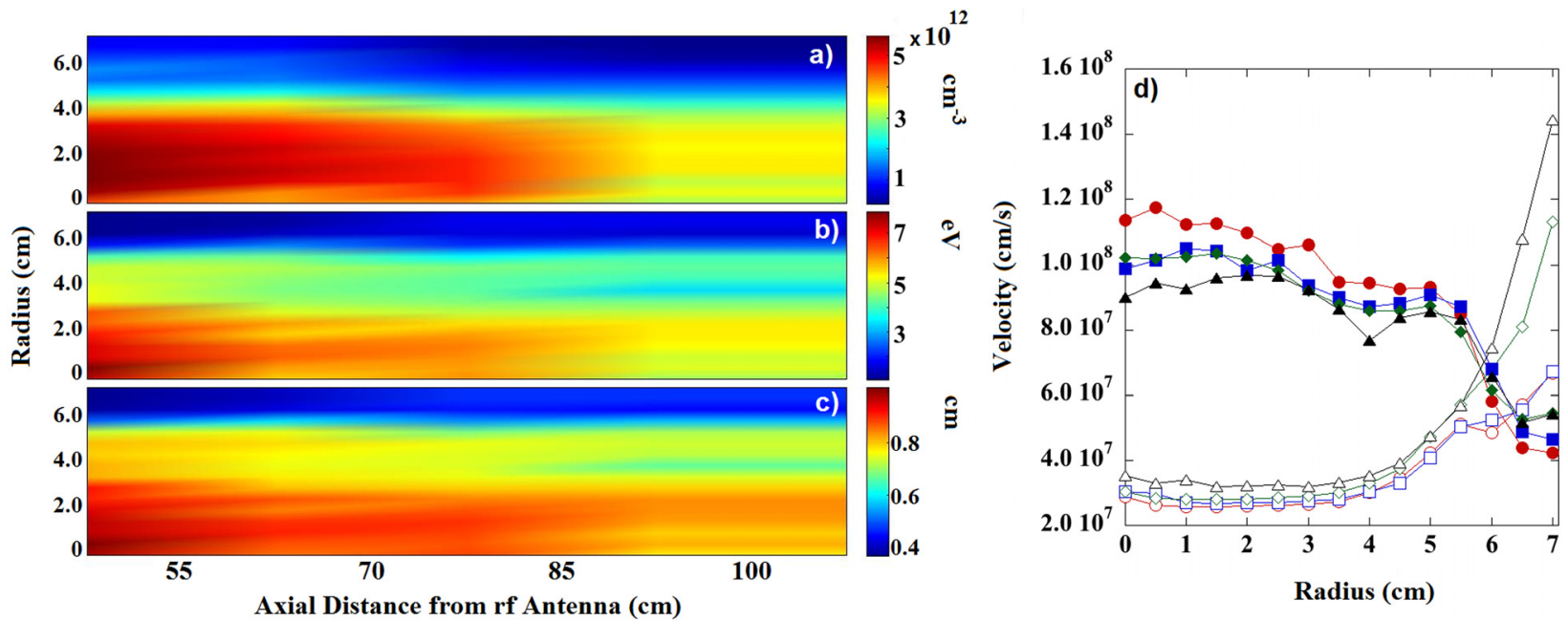

FIG. 3. (Color online) (a) Electron density, (b) electron temperature, (c) and ion sound gyroradius versus radius and axial distance from the rf antenna. (d) The electron thermal speeds (filled symbols) and Alfvén speeds (open symbols) as functions of plasma radius for different axial locations, at $z=55 \mathrm{~cm}$ (circles), $z=70$ $\mathrm{cm}$ (squares), $z=85 \mathrm{~cm}$ (diamonds), and $z=100 \mathrm{~cm}$ (triangles). The speeds are based on the density and temperature measurements shown in (a) and (b).

annulus. An ENI 1040L rf amplifier (driven with a HP 33120A function generator) supplies the current to the dipole antenna. The maximum magnetic field generated by the antenna is measured to be $12( \pm 2) \mathrm{G}$, over the frequency range $10-250 \mathrm{kHz}$. To avoid overheating of the dipole antenna, the helicon source is operated in a pulsed mode instead of its standard steady-state mode. Each helicon pulse lasts $256 \mathrm{~ms}$, and the pulse repetition frequency is $0.034 \mathrm{~Hz}$.

As a result of the helicon plasma creation process, there is considerable rf noise contamination of internal probe measurements in helicon sources. To suppress the rf noise in the experiments reported here, 100 differentially amplified time series (from either the electrostatic or magnetic probes) are synchronously averaged for each measurement. The averaging is synchronized with the initiation of the wave excitation by the dipole antenna. To eliminate the possibility that the background rf noise might also become synchronously averaged since the timing of the wave launching is based on a fixed delay from the start of the helicon pulse, a random time delay (shorter than a half-period of the rf wave) was added to the delay between the initiation of the helicon pulse and the wave excitation pulse for each of the 100 measurements. The

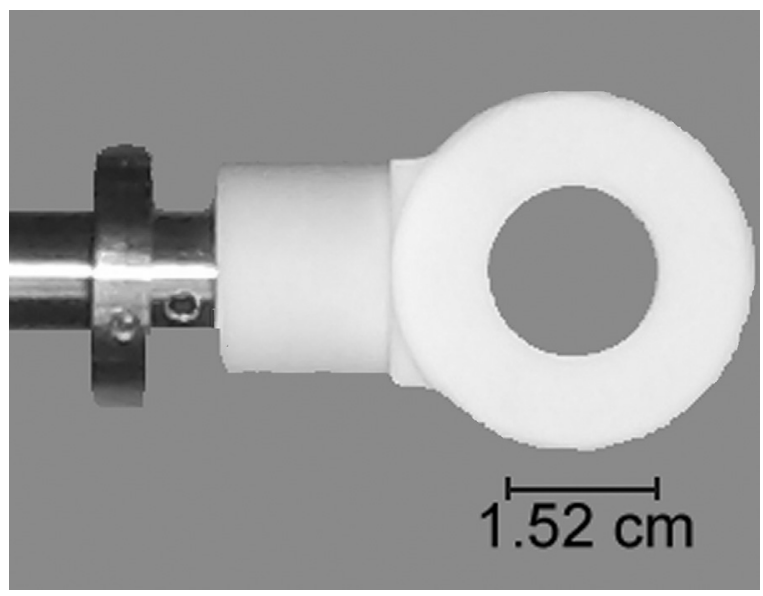

FIG. 4. The wave launching antenna assembly. average time series is post-processed with a narrow-band digital, zero-phase distortion, filter ${ }^{32}$ centered on the wave launching frequency. Although the digital filter does not play a particularly important role in the calculations of relative phase between signals from different probes, the reduction in noise obtained through the filtering process has a substantial effect on the calculation of the amplitudes of the launched waves from the probe measurements.

Each time series is recorded with a 12-bit Joerger VTR10012 digitizer. The digitizer has maximum digitation rate of $100 \mathrm{MHz}$ and each of the eight differential channels can acquire up to 262144 points. In the experiments reported here, the digitation rate was set to $1 \mathrm{MHz}$. Frequency aliasing of the $13.56 \mathrm{MHz}$ rf noise is eliminated by pre-processing the measured signals with a differential amplifier with a rolloff frequency well below the digitizer's Nyquist frequency of $500 \mathrm{kHz}$. Each measurement channel (pre-amplifier plus digitizer) has a small DC offset that is measured and subtracted out for the electrostatic and magnetic fluctuation measurements. The long record lengths of 262144 points for each measurement channel permit the subdivision of each time series measurement into smaller intervals to increase the effective number of data sets for averaging without significantly impacting the frequency resolution of each measurement. Thus, the random noise level in each averaged time series is further reduced by a factor of $M^{-1 / 2}$ for a set of $M$ time series.

\section{ELECTROSTATIC FLUCTUATION MEASUREMENTS}

A radially scanning 4-tip electrostatic probe is used to record electrostatic fluctuations during each wave launching pulse. The probe consists of four tungsten tips, $2 \mathrm{~mm}$ long and $0.59 \mathrm{~mm}$ in diameter, held in a 4-bore alumina shaft that is $4.8 \mathrm{~mm}$ in diameter. The alumina shaft extends $7.5 \mathrm{~cm}$ from a larger diameter boron nitride cap. Inside the probe shaft, the probe leads are enclosed in the alumina shaft and an additional thin copper tube to prevent pickup of electrostatic fluctuations along the length of the probe shaft. The 


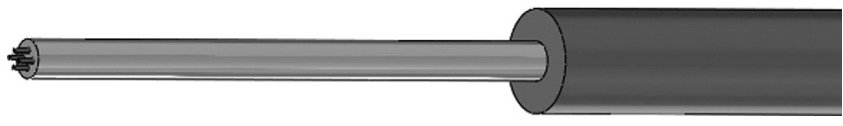

FIG. 5. Sketch of the electrostatic probe. The probe is inserted with the four tips ( $2 \mathrm{~mm}$ long and $0.59 \mathrm{~mm}$ in diameter) perpendicular to the magnetic field, with the upper and lower pairs aligned along the magnetic field. The alumina shaft extends $7.5 \mathrm{~cm}$ from the boron nitride housing. The top two tips or bottom two tips can be used for axial electric field $\left(E_{z}\right)$ measurements, and the two left two or two right tips can be used for azimuthal electric field $\left(E_{\theta}\right)$ measurements. The tungsten tips are spaced $1.4 \mathrm{~mm}$ apart.

tungsten tips are arranged in a square pattern with sides of $1.4 \mathrm{~mm}$ (see Fig. 5).

The electrostatic probe is inserted radially so that each two-tip pair is aligned along the background magnetic field. Measurements at different axial locations are obtained by physically moving the probe to a new port axial. The impact on the experimental system is minimal as the probe is inserted through a load-lock valve and chamber vacuum is maintained throughout the process. Each tip measures the floating potential fluctuations due to the waves launched from the dipole antenna. The average of the difference between the measurements from the top and bottom horizontally aligned pairs of tips is reported as the axial electric field $\left(E_{z}\right)$, which is proportional to the axial current driven by the antenna. The azimuthal electric field $\left(E_{\theta}\right)$ is calculated from the difference between the signals from the vertically aligned pair of tips closest to the antenna to minimize the effects of shadowing of the downstream tips on the azimuthal electric field measurement. The radial electric field $\left(E_{r}\right)$ is calculated from the differences between electric potential measurements using a single tip (the top tip closest to the antenna) obtained at multiple radial locations. For the radially resolved measurements, the probe is moved in $0.5 \mathrm{~cm}$ radial increments.

Shown in Fig. 6 is an example of 100 synchronously averaged potential fluctuation measurements for a $100 \mathrm{kHz}$ wave at $r=0 \mathrm{~cm}$ for three different axial locations: $70 \mathrm{~cm}$,
$85 \mathrm{~cm}$, and $100 \mathrm{~cm}$. The fluctuations are measured with the top tip closest to the antenna. Also shown in Fig. 6(d) are measurements at $z=70 \mathrm{~cm}$ as a function of radius. All time series shown have been post-processed with the digital filter described previously. The wave is easily visible in the measurements at $z=70 \mathrm{~cm}$ and $85 \mathrm{~cm}$. The radially resolved fluctuation observations clearly show that, $70 \mathrm{~cm}$ downstream of the antenna, the potential fluctuations arising from the wave pulses from the antenna are strongly confined to the central "core" of the plasma; $r<1 \mathrm{~cm}$. The radial extent of the large amplitude potential fluctuations is consistent with radial extent of the high density region of the plasma that can pass through the hole in the launching antenna. The two regions of reduced electrostatic perturbation amplitude that appear in Fig. 6 at $1.5-2.0 \mathrm{~cm}$ and at $4 \mathrm{~cm}$ roughly line up with the outer radius of the launching antenna and the transition to the metal shaft of the launching antenna probe. Thus, they appear to be artifacts of the wave excitation hardware.

The electric field amplitudes obtained from the difference in signal between two tips at $z=70 \mathrm{~cm}$ are shown in Fig. 7 as a function of radius for two different wave excitation frequencies: $50 \mathrm{kHz}$ and $100 \mathrm{kHz}$. Electric fields in all three cylindrical directions $(r, \theta, z)$ are observed with the radial electric field being the largest. The much larger radial electric field is shown on a different scale (left vertical axis of the plots) than the other two components (right vertical axis of the plots). Note that for the $50 \mathrm{kHz}$ excitation, the axial and azimuthal electric fields are limited to the inner few $\mathrm{cm}$ of the plasma. For the $100 \mathrm{kHz}$ wave, the electric field fluctuations extend to larger radii, with the significant radial electric field fluctuations out to $r=5 \mathrm{~cm}$. The appearance of any finite axial electric field and, thus, an axial current, is surprising given that the antenna is design to excite compressional Alfvén waves by inducing an azimuthal electric field in the plasma. In fact, the azimuthal electric field component is smaller than the axial electric field for all radii and for both
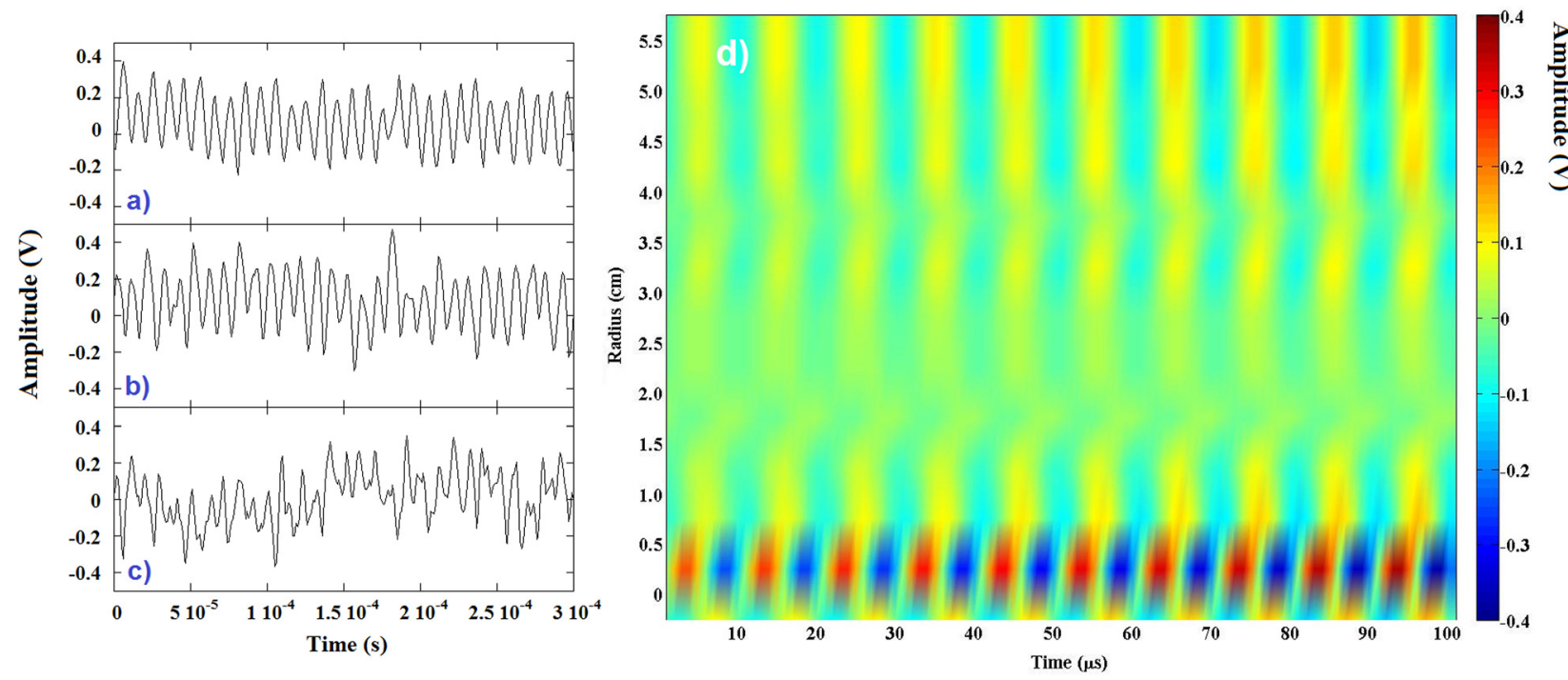

FIG. 6. (Color online) Synchronously averaged time series from the upper upstream electrostatic probe tip, for $r=0 \mathrm{~cm}$ and $100 \mathrm{kHz}$ at (a) $z=70 \mathrm{~cm}$, (b) $z=85 \mathrm{~cm}$, and (c) $z=100 \mathrm{~cm}$. (d) The synchronously averaged and digitally filtered electrostatic potential recorded by the digitizer from a single tip of the electrostatic probe at $z=70 \mathrm{~cm}$ versus radius and time. 

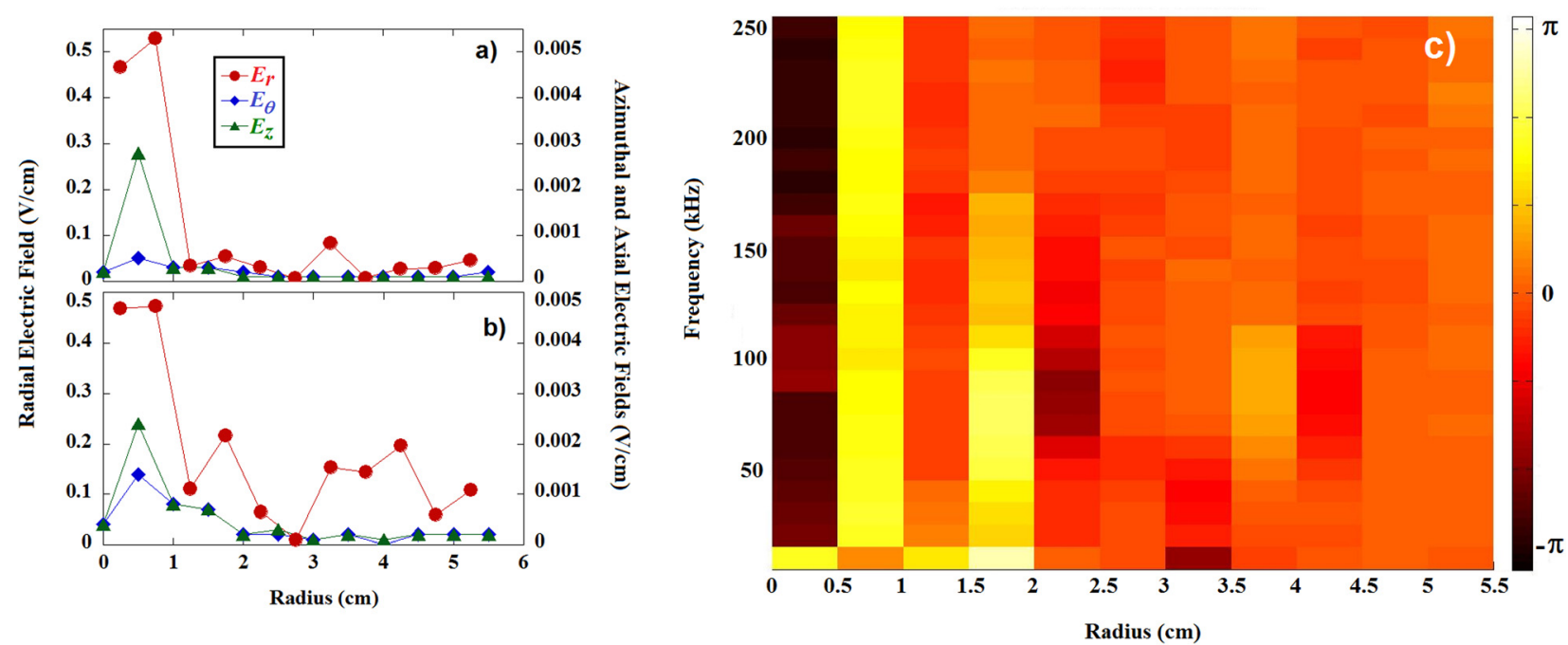

FIG. 7. (Color online) Amplitude of radial (circles), azimuthal (diamonds), and axial (triangles) electric field components measured at $z=70 \mathrm{~cm}$ for wave excitation frequencies of (a) $50 \mathrm{kHz}$ and (b) $100 \mathrm{kHz}$. (c) The radial phase difference obtained from radial electric field measurements as a function of plasma radius and wave frequency at $z=70 \mathrm{~cm}$.

wave frequencies examined. These measurements suggest that $70 \mathrm{~cm}$ downstream of the antenna, the wave excited by the antenna has coupled to a mode capable of driving shear Alfvén waves in the core region of the plasma.

Also shown in Fig. 7(c) are the radial phase differences obtained from the single probe tip as it is scanned across the plasma radius at $z=70 \mathrm{~cm}$. The floating potential fluctuations undergo a complete $2 \pi$ phase shift from $r=0$ to $r=1$ $\mathrm{cm}$. These phase shift measurements are consistent with electrostatic wave structures, i.e., perpendicular wavelengths, which are considerably smaller than twice the chamber radius at the $70 \mathrm{~cm}$ location.

\section{MAGNETIC FLUCTUATION MEASUREMENTS}

Two arrays of magnetic fluctuation sense coils (B-dot coils) are used to measure the phase velocity and amplitude of the Alfvén waves excited by the antenna. One probe contains an array of eight radially spaced $B_{r}$ coils. The other probe has an array of transverse coils that are used for $B_{\theta}$ and $B_{z}$ measurements. Each sense coil is wound from enameled 40 gauge wire on a cylindrical, ceramic bobbin. The eight coils are mated to the eight high-speed, differential, digitizer channels. As B-dot probes are prone to electrostatic fluctuation pickup, each probe array is inserted into a grounded 6.9 $\mathrm{cm}$ long, thin-walled stainless steel tube that is enclosed at one end. The other end is vacuum welded to a micro flange. The two leads of each coil pass through an additional 41.4 $\mathrm{cm}$ long stainless steel tube with another micro flange at one end and a grounded electronics box containing 8 floating BNC connectors at the other end. The micro flanges are sealed with a copper gasket and the entire coil array inserted into the vacuum chamber through a sliding vacuum seal. Thus, the B-dot coils are electrostatically shielded by the probe shaft and cooling air enters from the open end of the probe shaft. For the $B_{\theta}$ and $B_{z}$ measurements, the orientation of the internal coil array is held fixed relative to the probe shaft with an alignment pin.
Magnetic fluctuation measurements are performed at $z=70 \mathrm{~cm}, z=85 \mathrm{~cm}$, and $z=100 \mathrm{~cm}$ for the same wave launching antenna position of $z=55 \mathrm{~cm}$. An example of a synchronously averaged signal, where each signal is obtained from the first azimuthal coil, $B_{\theta}$, is shown in Fig. 8 for $r=3.2 \mathrm{~cm}$ and an excitation frequency of $100 \mathrm{kHz}$. Also shown in Fig. 8(d) are radially resolved $B_{\theta}$ measurements for the same wave excitation frequency at $z=85 \mathrm{~cm}$. An azimuthal fluctuation, a shear Alfvén wave, is clearly observed propagating in time in the radially resolved measurements. The wave amplitude is a maximum between radii of $2.5 \mathrm{~cm}$ and $4.0 \mathrm{~cm}$ and vanishes for radii larger than $4 \mathrm{~cm}$.

The magnetic fluctuation measurements at different axial and radial locations are post-processed to calculate the parallel wave number as a function of radius and wave excitation frequency, i.e., the dispersion relation. Only two spatially separated time series measurements are required to determine the parallel wave number. However, in this work, measurements were performed at three axial locations so that we could see if there is any evolution in the parallel wavelength as the wave propagated along the axis of the system. In fact, in the near field of the antenna, during comparison of magnetic fluctuation measurements from at $z=70 \mathrm{~cm}$ and $z=85 \mathrm{~cm}$, we find little evidence of shear Alfvén wave propagation. Further downstream, a clear and consistent phase difference along the axis is obtained for $B_{r}$ measurements at $z=85 \mathrm{~cm}$ and $z=100 \mathrm{~cm}$, at $r=0 \mathrm{~cm}$. The parallel wave number obtained from these measurements as a function of normalized wave frequency is shown in Fig. 9. Also shown in Fig. 9 is the theory curve for a kinetic Alfvén wave assuming a $\sim 7 \mathrm{~cm}$ perpendicular wavelength $\left(k_{\perp}=0.85 \mathrm{rad} / \mathrm{cm}\right)$ and including the collisional effects of the measured neutral helium density. As shown by Woods ${ }^{18}$ Alfvén wave fields must vanish at the boundary for a confined system. In cylindrical geometry, the wave fields are given by Bessel functions and this constraint is satisfied when the argument of the first Bessel function, $k_{\perp} a=3.83$, i.e., the first root. For a perpendicular wave number of $0.85 \mathrm{rad} / \mathrm{cm}$, the radius of the effective plasma 

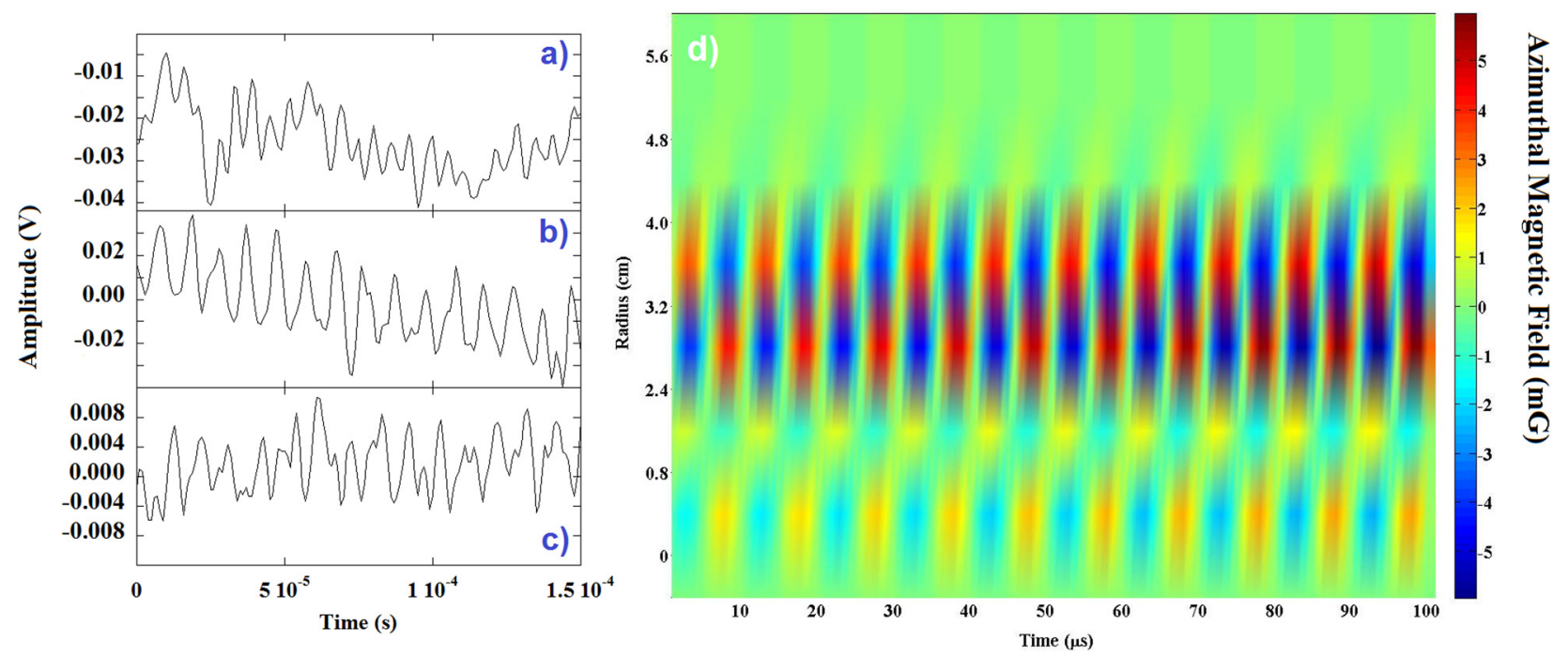

FIG. 8. (Color online) Synchronously averaged signal from the first azimuthal magnetic sense coil $\left(B_{\theta}\right)$ for a $100 \mathrm{kHz}$ wave measured at $r=3.2 \mathrm{~cm}$ and (a) $z=70 \mathrm{~cm}$, (b) $z=85 \mathrm{~cm}$, and (c) $z=100 \mathrm{~cm}$. (d) Synchronously averaged and digitally filtered averaged azimuthal magnetic field fluctuation signal versus time and radius at $z=85 \mathrm{~cm}$.

boundary is $4.5 \mathrm{~cm}$, the location of the boundary between the kinetic-inertial regimes of Alfvén waves. In other words, the perpendicular wave number obtained from the fit of theory to the measured dispersion is consistent with shear Alfvén wave propagation in a bounded cylinder of radius $4.5 \mathrm{~cm}$. Surprising to us is that we have not observed a significant compressional Alfvén wave component in our measurements. ${ }^{30}$ Classic Alfvén wave theory predicts that mode conversion from a compressional to a shear Alfvén wave should occur as a wave propagates from a low density to high density region. ${ }^{19}$ However, all of our wave excitation and subsequent dispersion relation measurements occur within the high den-

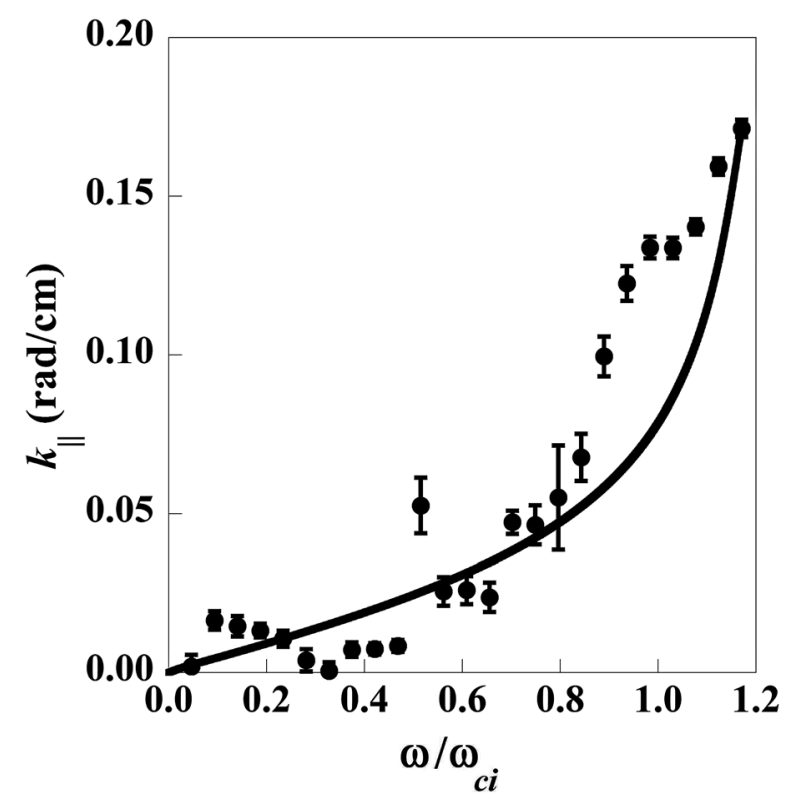

FIG. 9. The measured dispersion relation (circles) and the theoretical curve for a kinetic shear Alfvén wave assuming a magnetic field of $560 \mathrm{G}$, plasma density of $6 \times 10^{12} \mathrm{~cm}^{-3}$, neutral density of $5 \times 10^{12} \mathrm{~cm}^{-3}$, electron temperature of $7 \mathrm{eV}$, ion temperature of $0.25 \mathrm{eV}$, ion-sound gyroradius of $0.8 \mathrm{~cm} / \mathrm{rad}$, and perpendicular wave vector of $0.85 \mathrm{rad} / \mathrm{cm}$. sity region of our plasma. We hypothesize that compressional waves, if they are directly excited by the antenna, propagate outwards radially and upon reflection at the inertial-kinetic boundary, couple to shear Alfvén waves as the reflected wave propagates back into the higher density plasma core. Without measurements very close to the antenna, however, this hypothesis cannot be tested experimentally. We are currently engaged in developing computational models that may be able to address this important question.

The density and electron temperature profiles in these plasmas naturally divide the plasma into regions of kinetic and inertial Alfvén wave propagation. As shown in Fig. 3, this division occurs at a radius of approximately $4-5 \mathrm{~cm}$. In the measurements shown in Fig. 8(d), there is a dramatic reduction in Alfvén wave power at roughly the same radius. In previous experiments in the LAPD device, Alfvén wave energy was observed to "accumulate" at the inertial-kinetic wave boundary where the perpendicular group velocity went to zero. ${ }^{11}$ The energy stored in a shear Alfvén wave is calculable from the integral expression

$$
U_{\theta}=\frac{1}{8 \pi} \int_{0}^{2 \pi}\left\langle B_{\theta}\right\rangle^{2} r d \theta \approx r\left\langle B_{\theta}\right\rangle^{2} .
$$

Shown in Fig. 10 is the stored energy versus radius according to Eq. (14) for a $100 \mathrm{kHz}$ wave. Consistent with the data shown in Figure 8, the wave stored energy is a maximum for radii just inside of the kinetic-inertial boundary.

Identifying the $x$ and $z$ axes in Eq. (4) as the directions perpendicular and parallel to the background magnetic field, the group velocity of a kinetic Alfvén wave is obtained from the dielectric tensor components

$$
n_{\perp}^{2} \varepsilon_{\perp}+n_{\|}^{2} \varepsilon_{\|}=\varepsilon_{\perp} \varepsilon_{\|},
$$

where $\mathbf{n}=c \mathbf{k} / \omega$, is the index of refraction and $\boldsymbol{\varepsilon}$ is the dielectric tensor. Solving Eq. (16) for the perpendicular group velocity 


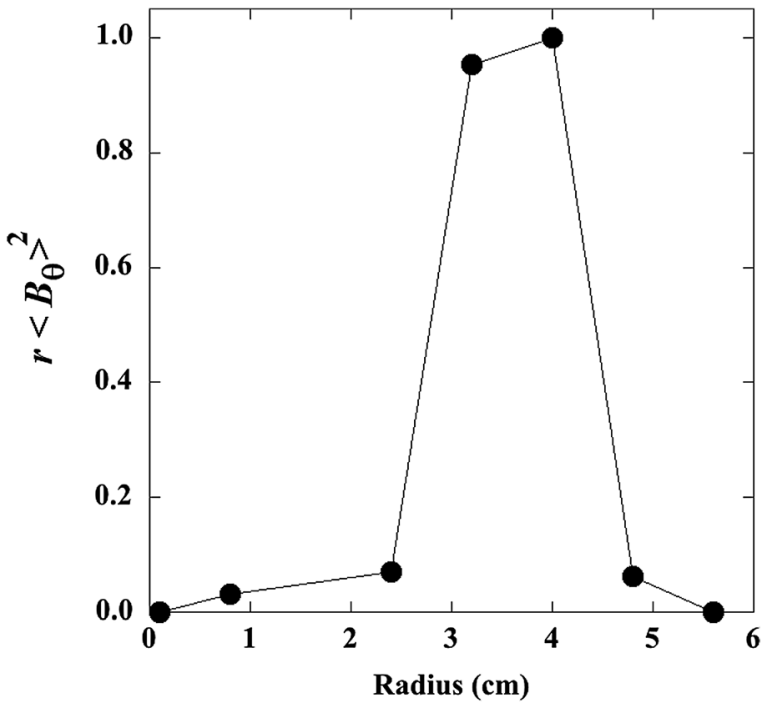

FIG. 10. Stored wave energy based on the azimuthal magnetic fluctuation measurements as a function of plasma radius for wave excitation frequency of $100 \mathrm{kHz}$. These measurements are performed at $\mathrm{z}=100 \mathrm{~cm}$ and are normalized to the peak value.

$$
\mathrm{v}_{g \perp}=2 k_{\perp}\left[\left(\frac{\omega^{2}}{c^{2}}-\frac{k_{\|}^{2}}{\varepsilon_{\perp}}\right) \frac{\partial \varepsilon_{\|}}{\partial \omega}+\varepsilon_{\|}\left(\frac{2 \omega}{c^{2}}+\frac{k_{\|}^{2}}{\varepsilon_{\perp}^{2}} \frac{\partial \varepsilon_{\perp}}{\partial \omega}\right)\right]^{-1}
$$

where the perpendicular and parallel components of the dielectric tensor are given in Eqs. (5) and (6). Inserting the shear Alfvén wave dispersion relation, Eq. (17) reduces to the complex expression ${ }^{11}$

$$
\mathrm{v}_{g \perp}=-2 k_{\perp} \rho_{s}^{2} \omega / Z^{\prime}(\zeta)
$$

where $Z^{\prime}$ is the derivative of the plasma dispersion function and $\zeta=\omega / k_{\|} \mathrm{v}_{\mathrm{e}}$ is the normalized wave phase speed. Following the analysis of Vincena et al. ${ }^{11}$ and Stratton, ${ }^{33}$ it is the real part of $Z^{\prime}$ that is physically relevant and the group velocity becomes

$$
\mathrm{v}_{g \perp}=-2 k_{\perp} \rho_{s}^{2} \omega \operatorname{Re}\left[Z^{\prime}(\zeta)\right] /\left|Z^{\prime}(\zeta)\right|^{2} .
$$

The real part of $Z^{\prime}$ goes to zero, and thus, the group velocity goes to zero, where $\zeta \approx 0.924$. Using the measured plasma and wave parameters, the calculated value of $\zeta$ is shown in Fig. 11 as a function of plasma radius for a variety of wave frequencies. The parallel wave numbers used for the $\zeta$ data are based on the magnetic fluctuation measurements at $z=85 \mathrm{~cm}$ and $z=100 \mathrm{~cm}$. Thus, at the boundary separating the kinetic and inertial Alfvén regimes, theory combined with the measured parallel wavelengths yields a prediction that the perpendicular wave group velocity should approach zero, thereby "ducting" the Alfvén wave within the uniform plasma region in the same way an optical fiber achieves total internal reflection. The observed increase in stored wave energy around radii of $4 \mathrm{~cm}$, just inside the boundary at radii of 5-6 cm evident in Fig. 11, is consistent with Alfvén wave ducting within the high density core plasma.

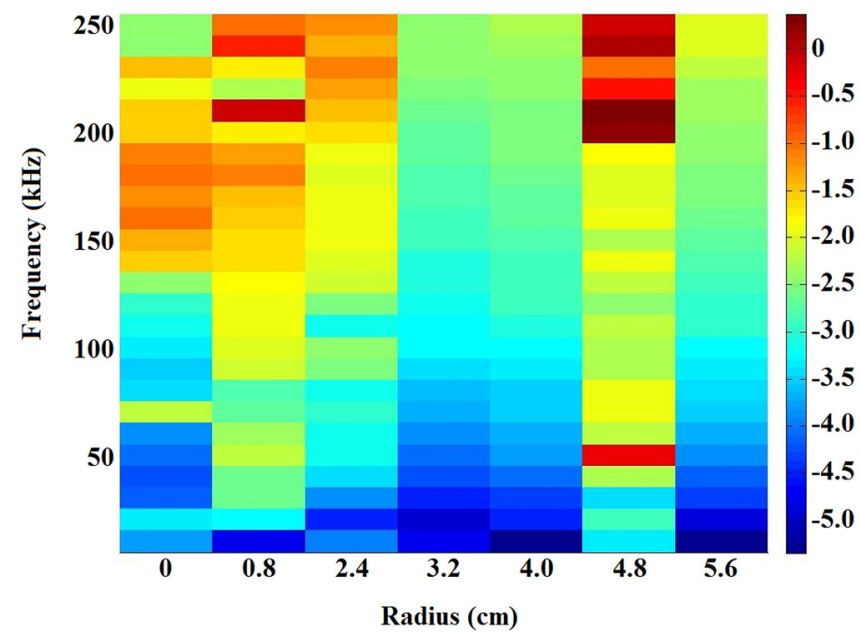

FIG. 11. (Color online) The logarithm of $\zeta\left(=\omega / k_{\|} \mathrm{v}_{\mathrm{e}}\right)$ as a function of plasma radius and wave excitation frequency. The wave phase difference measurements used are based on the measurements obtained between axial positions of $z=85 \mathrm{~cm}$ and $z=100 \mathrm{~cm}$. At $r=4.8 \mathrm{~cm}$, the same location as the boundary between kinetic and inertial regimes, $\zeta$ has local maxima for each driving frequency.

\section{SUMMARY}

The experimental observations presented here are consistent with a shear kinetic Alfvén waves being ducted inside the higher density, core, region of a helium helicon plasma. Features of this work are similar to a previous study of Alfvén wave propagation in a helicon source. ${ }^{15}$ However, in these experiments, the neutral helium density was directly measured, the high density core of the plasma clearly plays a key role in the wave dispersion, and the launching antenna was designed to couple to compressional and not shear Alfvén waves. At the radius where the Alfvén speed and the electron thermal speed are equal, the perpendicular group velocity appears to decrease and the wave energy accumulates-consistent with previous experiments in LAPD.

These experiments demonstrate that Alfvén wave experiments are feasible in any high-density helicon source. However, since the long-term goal of these Alfvén wave experiments is to understand the ion heating mechanisms in the Sun's corona, future experiments will involve the excitation of oppositely propagating Alfvén waves at larger amplitudes so that nonlinear cascades to smaller spatial scales might be observed. Additional control of the perpendicular wavelength of the excited waves, a key parameter in the nonlinear coupling process, might be achievable through the development of launching structures with enforced, small scale, periodic structures. ${ }^{34}$

\section{ACKNOWLEDGMENTS}

This work was supported by NSF Award No. PHY0902085.

${ }^{1}$ R. Erdélyi and V. Fedun, Scinece 318, 1572 (2007).

${ }^{2}$ J.-S. He, C.-Y. Tu, E. Marsch, L.-J. Guo, S. Yao, and H. Tian, Astron. Astrophys. 497, 525 (2009).

${ }^{3}$ J. W. Belcher, Astrophys. J. 168, 509 (1971). 
${ }^{4}$ J. V. Hollweg, J. Geophys. Res. 105(A7), 15699, doi:10.1029/ 1999JA000449 (2000).

${ }^{5}$ C.-Y. Tu and E. Marsch, J. Geophys. Res. 106, 8233, doi:10.1029/ 2000JA000024 (2001).

${ }^{6}$ J. V. Hollweg and P. A. Isenberg J. Geophys. Res. 107(A7), 1147, doi:1029/2001JA000270 (2002).

${ }^{7}$ T. K. Suzuki and S. Inutsuka, Astrophys. J. Lett. 1, L49 (2005).

${ }^{8}$ V. Angelopoulos, J. P. McFadden, D. Larson, C. W. Carlson, S. B. Mende, H. Frey, T. Phan, D. G. Sibeck, K.-H. Glassmeier, U. Auster, E. Donovan, I. R. Mann, I. J. Rae, C. T. Russell, A. Runov, X.-Z. Zhou, and L. Kepko, Science 321, 931 (2008).

${ }^{9}$ D. J. Knudsen, J. Geophys. Res. 101, 10761, doi:10.1029/96JA00429 (1996).

${ }^{10}$ H. Alfvén, Nature 150, 405 (1942).

${ }^{11}$ S. Vincena, W. Gekelman, and J. Maggs, Phys. Rev. Lett. 93, 105003 (2004).

${ }^{12}$ W. Gekelman, H. Pfister, Z. Lucky, J. Bamber, D. Leneman, and J. Maggs, Rev. Sci. Instrum. 62, 2875 (1991).

${ }^{13}$ Y. Amagishi, M. Inutake, T. Akitsu, and A. Tsushima, Jpn. J. Appl. Phys. 20, 2127 (1981).

${ }^{14}$ Y. Amagishi and A. Tsushima, Plasma. Phys. Controlled Fusion 26, 1489 (1984).

${ }^{15}$ C. Watts and J. Hanna, Phys. Plasmas 11, 1358 (2004).

${ }^{16}$ O. Grulke, C. M. Franck, and T. Klinger, AIP Conf. Proc. 669, 412 (2003).

${ }^{17}$ W. Gekelman, S. Vincena, D. Leneman, and J. Maggs, J. Geophys. Res. 102, 7225, doi:10.1029/96JA03683 (1997).

${ }^{18}$ L. C. Woods, J. Fluid. Mech. 13, 570 (1962).
${ }^{19}$ A. Hasegawa and C. Uberoi, The Alfvén Waves (Technical Information Center, U.S. Department of Energy, USA, 1982).

${ }^{20}$ K. Stasiewicz, P. Bellan, C. Chaston, C. Kletzing, R. Lysak, J. Maggs, P. Pokhotelov, C. Seyler, P. Shukla, L. Stenflo, A. Streltsov, and J.-E. Wahland, Space. Sci. Rev. 92, 423 (2000).

${ }^{21}$ D. G. Swanson, Plasma Kinetic Theory (CRC, Taylor \& Francis Group, Boca Raton, FL, 2008).

${ }^{22}$ R. L. Lysak and W. Lotko, J. Geophys. Res. 101, 5085, doi:10.1029/ 95JA03712 (1996).

${ }^{23}$ R. A. Koch and W. Horton, Jr., Phys. Fluids 18, 861 (1975).

${ }^{24}$ G. Müller, Plasma Phys. 16, 813 (1974).

${ }^{25}$ D. G. Swanson, Plasma Waves, 2nd ed. (IOP, Bristol, UK, 2003).

${ }^{26}$ S. Yun, K. Taylor, and G. R. Tynan, Phys. Plasmas 7, 3448 (2000).

${ }^{27}$ Y. Amagishi and M. Tanaka, Phys. Rev. Lett. 71, 360 (1993).

${ }^{28}$ J. L. Kline, E. E. Scime, R. F. Boivin, A. M. Keesee, and X. Sun, Plasma Sources Sci. Technol. 11, 413 (2002).

${ }^{29}$ S. Houshmandyar, S. Sears, S. Chakraborty Thakur, J. Carr, Jr., M. Galante, and E. E. Scime, Rev. Sci. Instrum. 81, 10D704 (2010).

${ }^{30}$ S. Houshmandyar, Ph.D. thesis, Department of Physics, West Virginia University, 2010.

${ }^{31}$ S. Houshmandyar and E. E. Scime, Plasma Sources Sci. Technol. (to be published).

${ }^{32}$ F. Gustafsson, IEEE Trans. Signal Process. 44, 988 (1996).

${ }^{33}$ J. A. Stratton, Electromagnetic Theory (McGraw-Hill, New York, 1941).

${ }^{34}$ C. A. Kletzing, S. R. Bounds, and J. Martin-Hinder, Phys. Rev. Lett. 90, 035004 (2003). 